\title{
Gene expression during the differentiation of bone marrow stem cells into cardiomyocytes
}

\author{
Xiaohong $\mathrm{Li}^{1}$, Xiyong $\mathrm{Yu}^{1}$, Zhixin Shan ${ }^{1}$, Qiuxiong $\mathrm{Lin}^{1}$, Jiuchang Zhong ${ }^{1}$, Yongheng $\mathrm{Fu}^{1}$, Wei Huang ${ }^{1}$, Min \\ Yang $^{1}$, Shuguang Lin $^{1}$ \\ ${ }^{I}$ Research center of Medical Sciences, Guangdong Province People's Hospital, Guangdong Provincial Cardiovascular Institute. \\ Guangzhou 510080, China
}

Heart attacks and congestive heart failure remain among the world's most prominent health challenges. Though there are many breakthroughs in heart disease treatment, myocardial infarction (MI) result in irreversible damage that functional cardiomyocytes have been lost. Cell transplantation has emerged as a potential new approach for repairing damaged myocardium. In previous study, we have found Bone marrow mesenchymal stem cells (BMSCs) can transdifferentiate into myocardial cell. In this study we focused to investigate molecular regulation mechanism of promoting BMSCs to differentiate into myocardial cell phenotype, and to provide the base condition on large scale transforming BMSCs into cardiomyocytes. BMSCs were isolated and purified from bone marrow of clinical patients by $1.073 \mathrm{~g} / \mathrm{ml}$ density gradient centrifugation and adherence ability. Cells were expanded as undifferentiated cells in culture for more than 3 passages and cocultured with neonatal rat ventricular myocytes in a rate of 1:10 separated by semipermeable membrane. After cocultured 3 days, 2 weeks, and 8 weeks separately, gene expression profile changes were analyzed by gene chip and different expression level of cardiac myogenesis related genes were presented by Cluster analysis. BMSCs differentiation related gene time series were detected before and after coculture. Heartspecific primary microRNAs (primiRNAs) in the transformed cardiomyocyte cells were detected by RT-PCR and contrasted with 5-aza induced BMSCs. Gene differential expression arrays demonstrated that there were 45, 511 and 569 genes upregulated (ratio $>5$ ). However, there were 57, 336 and 1874 genes down-regulated (ratio $<0.2)$. Genes about development, myogenesis and anti-apoptosis were upregulated. The time course of myogenesis genes showed that HOP, GATA4, NKx2.5, $\beta$-MHC and ANF mRNA were increased, and expressed maximum in two weeks. miRNA$143,-181$ could be induced to express by 5 -azacytidine and miRNA-143, $-181,-206,-208$ could be induced to express by coculturation with cardiomyocytes. Therefore, the differentiating process of BMSCs was regulated by multiple factors. Such as HOP, Nkx2.5, and GATA4 genes expressed in a time course and copromoted myogenesis. Heartspecific microRNAs involved in the process of cardiomyocyte differentiation, and that induction of these microRNAs may be important in regulating the expression of heart-specific proteins.

Keywords: marrow mesenchymal stem cell, differentiation, cardiomyocyte

Cell Research (2008) 18:s52. doi: 10.1038/cr.2008.142; published online 4 August 2008

Correspondence: Xiyong Yu

E-mail: yuxycn@163.com

Supported by National Natural Science Foundation of China (30672077). 